\title{
AN EXTENSION OF KHINTCHINE'S INEQUALITY ${ }^{1}$
}

\author{
BY C. M. NEWMAN
}

Communicated March 20, 1975

Khintchine's inequality [4] states that if $\left\{X_{j}: j=1, \ldots, N\right\}$ are independent identically distributed Bernoulli random variables $\left(X_{j}= \pm 1\right.$ with equal probabilities), then for any choice of real $a_{j}$, and any $m=2,3, \ldots, X=\Sigma_{j} a_{j} X_{j}$ satisfies

$$
E\left(X^{2 m}\right) \leqslant\left((2 m) ! / 2^{m} m !\right)\left(E\left(X^{2}\right)\right)^{m} .
$$

This inequality implies [9, Chapter 5] that for $0<p<\infty$, there exist positive constants $A_{p}$ and $B_{p}$ depending only on $p$ (with $B_{2 m}=\left((2 m) ! / 2^{m} m !\right)^{1 / 2 m}$ ) such that

$$
A_{p}\|X\|_{2} \leqslant\|X\|_{p} \leqslant B_{p}\|X\|_{2}
$$

where $\|X\|_{p}$ denotes the $p$-norm, $\left(E\left(|X|^{p}\right)\right)^{1 / p}$. Khintchine's inequality in this form has many applications in which the $\left\{X_{j}\right\}$ are generally represented as Rademacher functions [9], [7], [3] .

In this note we give an extension of Khintchine's inequality from the Bernoulli case to that of random variables of the following type:

Definition. A random variable $X$ is of type $L$ if its moment generating function $E_{X}(z) \equiv E(\exp (z X))$ satisfies

(i) $\exists C \ni E_{X}(z) \leqslant \exp \left(C z^{2}\right)$ for all real $z$ and

(ii) $E_{X}(z)=0 \Rightarrow z=i \alpha$ for some real $\alpha$.

Symmetric random variables satisfying condition (i) have been called subgaussian by Kahane; they satisfy an inequality similar to but weaker than (1) [2, p. 87].

Theorem 1 below extends Khintchine's inequality to arbitrary linear combinations of independent random variables of type $L$ while Theorem 2 treats the case of positive linear combinations of type $L$ random variables with a particular kind of dependence (such as arises in models of ferromagnets). Complete proofs of these theorems together with further results concerning random variables of type $L$ and applications of these results to statistical mechanics and quantum field theory will appear in $[6]{ }^{2}$

THEOREM 1. If $\left\{X_{j}\right\}_{j=1}^{N}$ are independent (not necessarily identically distributed) random variables of type $L$, then the inequality (1) applies for any

AMS (MOS) subject classifications (1970). Primary 42A36, 60G50; Secondary 42 A56.

${ }_{2}^{1}$ Supported in part by NSF MPS 74-04870.

2 Other related results are contained in a paper, Gaussian correlation inequalities for ferromagnets, by the author, which will appear in $\mathrm{Z}$. Wahrscheinlichkeitstheorie und Verw. Gebiete. 
choice of real $a_{j}$ and any $m=2,3, \ldots$ to $X=\Sigma_{j} a_{j} X_{j}$.

SKETCH OF PROOF. Since the $X_{j}$ are independent, $X$ is itself of type $L$. Hadamard factorization methods [1, Theorems 2.7.1 and 2.10.1] imply that for any random variable $X$ of type $L$,

$$
E_{X}(z)=\exp \left(b z^{2}\right) \prod_{j}\left(1+\left(z / \alpha_{j}\right)^{2}\right)
$$

for some $b \geqslant 0$ and $0<\alpha_{1} \leqslant \alpha_{2} \leqslant \cdots$ with $\Sigma\left(1 / \alpha_{j}\right)^{2}<\infty$. We next note that $E_{X}(z)=\Sigma E\left(X^{n}\right) z^{n} / n$ ! so that by (3),

$$
E\left(X^{2}\right) / 2=b+\sum_{j}\left(1 / \alpha_{j}\right)^{2} .
$$

Now each Taylor coefficient of $\left(1+\left(z / \alpha_{j}\right)^{2}\right)$ is bounded by the corresponding Taylor coefficient of $\exp \left(\left(z / \alpha_{j}\right)^{2}\right)$ from which it follows by (3) and (4) that each Taylor coefficient of $E_{X}(z)$ is bounded by the corresponding one of $\exp \left(z^{2} E\left(X^{2}\right) / 2\right)$ which yields (1).

REMARK. $X$ may satisfy (1) without being of type $L$ as can be seen by considering the probability distribution

$$
(1-\beta) \delta(x)+\beta(\delta(x-1)+\delta(x+1)) / 2 \text { for } 1 / 3 \leqslant \beta<1 / 2 .
$$

THEOREM 2. Suppose $\left\{Y_{j}\right\}_{j=1}^{N}$ are random variables whose joint probability distribution $\rho$ on $\mathbf{R}^{N}$ is of the form

$$
\rho\left(y_{1}, \ldots, y_{N}\right)=C^{\prime} \exp \left(\sum_{j, k=1}^{N} J_{j k} y_{j} y_{k}\right) \prod_{j=1}^{N} \mu_{j}\left(y_{j}\right),
$$

with $J_{j k} \geqslant 0 \forall j, k$, and with each $\mu_{j}$ an even measure satisfying:

(a) $\int \exp \left(b y^{2}\right) d \mu_{j}(y)<\infty \quad \forall b>0$, and

(b) $\int \exp (z y) d \mu_{j}(y)=0 \Rightarrow z=i \alpha$ for some real $\alpha$;

then for any choice of $\lambda_{j} \geqslant 0, X \equiv \Sigma \lambda_{j} Y_{j}$ is of type $L$ and thus satisfies (1) $(m=2,3, \ldots)$.

SKETCH OF PROOF. Theorem 2 follows directly from the proof of Theorem 1 combined with a general version of the (Statistical Mechanics) Lee-Yang Theorem [5, Theorem 1.1].

Examples of measures $\mu$ satisfying (a) and (b) ${ }^{3}$ (and thus examples of type $L$ random variables) include:

$$
\begin{gathered}
\mu(y)=\sum_{k=0}^{n} \delta(y-(n-2 k)), \quad n=1,2, \ldots \\
d \mu / d y=\left\{\begin{array}{ll}
1, & |y| \leqslant A, \\
0, & |y|>A,
\end{array} \quad A>0,\right.
\end{gathered}
$$

${ }^{3}$ Many other examples can be found in various of Polya's papers on the location of zeros of entire functions. 


$$
d \mu / d y=\left\{\begin{array}{ll}
\left(1-y^{2}\right)^{(d-2) / 2}, & |y| \leqslant 1, \\
0, & |y|>1,
\end{array} \quad d>0\right.
$$

$$
d \mu / d y=\exp (-\lambda \cosh y), \quad \lambda>0
$$

$$
d \mu / d y=\exp \left(-a y^{4}-b y^{2}\right), \quad a>0 \quad[8]
$$

When $d$ is an integer, example (8) is the one-dimensional marginal distribution of the uniform distribution on the surface of the unit $d$-sphere (in $\mathbf{R}^{d+1}$ ).

\section{REFERENCES}

1. R. P. Boas, Jr., Entire functions, Academic Press, New York, 1954. MR 16, 914.

2. J. P. Kahane, Séries de Fourier aléatoires, Deuxième édition multigraphiée, Les Presses de 1'Université de Montréal, Montreal, 1967. MR 42 \#3483.

3. T. Kawata, Fourier analysis in probability theory, Academic Press, New York, 1972.

4. A. Khintchine, Über dyadische Brüche, Math. Z. 18 (1923), 109-116.

5. C. M. Newman, Zeros of the partition function for generalized Ising systems, Comm. Pure Appl. Math. 27 (1974), 143-159.

6. - Inequalities for Ising models and field theories which obey the Lee-Yang theorem, Comm. Math. Phys. 41 (1975), 1-9.

7. A. Pelczyński, $A$ characterization of Hilbert-Schmidt operators, Studia Math. 28 (1966/67), 355-360. MR 35 \#7163.

8. B. Simon and R. B. Griffiths, The $\left(\phi^{4}\right)_{2}$ field theory as a classical Ising model, Comm. Math. Phys. 33 (1973), 145-164.

9. A. Zygmund, Trigonometric series. Vol. I, 2nd ed., Cambridge Univ. Press, New York, 1959. MR 21 \#6498.

DEPARTMENT OF MATHEMATICS, INDIANA UNIVERSITY, BLOOMINGTON, INDIANA 47401 